\title{
Construct validation of the English version of Readiness for Interprofessional Learning Scale (RIPLS): Are Chinese undergraduate students ready for shared learning?
}

\begin{abstract}
Interprofessional education (IPE) has been receiving attention as a result of research suggesting the benefits of interpersonal collaboration in health care. In Hong Kong, the implementation of the Interprofessional Team-based Learning (IPTBL) programme provides implicit call to study the psychometric properties of Readiness for Interprofessional Learning Scale (RIPLS) to clarify if this is a valid measure when used in the Chinese undergraduate healthcare context. This study examines the psychometric properties of RIPLS involving predominantly Chinese undergraduate healthcare students in Hong Kong. Using within- and between-network approaches to construct validity, we investigated the applicability of English Version of RIPLS among 469 predominantly Hong Kong Chinese students who have competence in the English language. These participants were from complementary health professional programs: Biomedical Sciences, Chinese Medicine, Medicine, Nursing, and Pharmacy, from two universities in Hong Kong. The withinnetwork test results indicated that RIPLS had good internal consistency reliability. Results of the confirmatory factor analysis (CFA) lend support to the overall factor structure of hypothesized four-factor solution although one item obtained non-significant factor loading. The betweennetwork test also suggests that various subscales of RIPLS correlated systematically with theoretically relevant constructs: collective efficacy, team impact on quality of learning, and team impact on clinical reasoning ability. The RIPLS is a valid measure to estimate the Chinese undergraduate healthcare students’ readiness to engage in interprofessional learning.
\end{abstract}

Keywords: Interprofessional education, RIPLS, construct validity, within-network construct validity, between network-construct validity

\section{Introduction}

The momentum for interprofessional education (IPE) is taking place in healthcare and social service professionals because of the recognition of the enormous advantages of interprofessional management of patients. IPE, which occurs when individuals from two or more professions learn with, about, and from each other (Barr \& Low, 2013), is linked closely with improved professional practice and patients' satisfaction of health treatment and care (Reeves, 
Perrier, Goldman, Freeth, Zwarenstein, 2013). Thus, IPE and collaborative practice, which connotes that no single profession can optimize patient care, are perceived to be beneficial in providing quality patient care (National Research Council, 2003; Reeves, et al., 2013). To prepare the students for the interprofessional management of patients, it is interesting to note that curricula are refined to include authentic learning activities to trigger the development of students' collaboration and teamwork in the care and delivery of health services to patients.

In Hong Kong, a large-scale IPE has been implemented involving a large number of healthcare and social service students from two government funded universities (Chan \& Ganotice, 2015a, b). This development necessitates preparation of assessment tools critical in understanding the readiness of students for interprofessional education. This is especially important on the assumption that students' attitude towards group work is substantially dependent on their attitude and readiness for shared learning (Gilbert, 2005; Parsell \& Bligh, 1999).

One of the scales that is popularly used in IPE is the Readiness for Interprofessional Learning Scale (RIPLS, Parsell \& Bligh, 1999). Despite the available translation and validation studies (e.g.,Tamaru et al., 2012; Tyastuti, Onishi, Ekayanti \& Kitamura, 2014), to the best of our knowledge, there has been no report on the validity of RIPLS in the Chinese context. Validation is an important preliminary step when used in a new setting that enables comparisons of results across different studies and contexts (Hambleton, 2001; Maneesriwongul \& Dixon, 2004). The establishment of cultural fit of the instruments is necessary before a sound interpretation can be made. This study therefore attempts to clarify the acceptability of RIPLS involving Chinese undergraduate healthcare students in Hong Kong. Specifically, this study purports to (a) examine the extent to which the RIPLS four-factor solution which has been established in previous 
research, emerges in a predominatly Hong Kong Chinese sample; and (b) clarify how the four factors in the RIPLS correlate with theoretically relevant constructs.

\section{Readiness for Interprofessional Learning Scale (RIPLS)}

The RIPLS (Parsell \& Bligh, 1999), a self-report questionnaire, is designed to estimate the degree of readiness of students to engage in interprofessional learning activities. The original 19 items represent the three IPE readiness factors: teamwork and collaboration, professional identity, and roles and responsibilities. The subsequent validation studies (i.e., McFadyen, et al., (2005) provided a possible more stable model different from the original version where the subscale "professional identity” was bifurcated into subscales: positive professional identity and negative professional identity. The scale is designed to be answered in a 5-point Likert scale from 1 (strongly disagree) to 5 (strongly agree) where higher values demonstrate the presence of the construct (e.g., professional identity).

Parsell and Bligh validated the RIPLS (Parsell \& Bligh, 1999) which has become a popular measure in determining the interprofessional readiness of students. This stimulated other researchers to clarify the validity of RIPLS in different contexts. However, validation studies did not yield similar findings. While a number of studies supported the two-factor to three-factor solutions (e.g., Lauffs et al., 2008), others obtained data which supported four-factor solutions, and some others obtained different findings (McFadyen, Webster, \& Maclaren, 2006; McFadyen et al., 2005; Tamura et al., 2012). These inconsistent findings suggest that further work is necessary to shed light on the psychometric acceptability of RIPLS especially when this is used in other cultures.

The RIPLS has been validated and adapted into different languages such as Swedish (Lauffs et al., 2008), Indonesian (Tyastuti et al., 2014), Japanese (Tamaru et al., 2012), German (Mahler 
et al., 2016), French (Cloutier, Lafrance, Michallet, Marcoux \& Cloutier, 2015), and Danish (Nørgaard, Draborg \& Sørensen, 2016). While we recognized the importance of translating the RIPLS into Chinese language, we were also mindful that healthcare students in Hong Kong have attained a certain degree of English proficiency for admission to any undergraduate programs. We therefore validated the original 19-item English version of RIPLS.

Students' team experience and collective efficacy

Students’ readiness to engage in interprofessional learning is conceptually linked with collective team efficacy, overall satisfaction with team experience, and team impact on team experience, and team impact on clinical reasoning ability. For example, various studies demonstrate the positive relationship between working in teams (e.g., teamwork and collaboration) and students’ quality of learning, and between collective efficacy and achievement (e.g., Johnson \& Johnson, 1999; Oyarzun \& Morrison, 2013; Salanova, Llorens, Cifre, Martínez \& Schaufeli, 2003). Thus, we hypothesized that the positive factors of RIPLS (e.g., teamwork and collaboration, positive professional identity, and roles and responsibilities) would be associated positively with a number of theoretically-relevant variables: collective team efficacy, team impact on quality of learning, and team impact on clinical reasoning; whereas the only negative factor of RIPLS (e.g., negative professional identity) would be associated negatively with the said theoretically-relevant variables.

\section{Methods}

\section{Construct Validation}

We adopted the construct validation approach (Marsh, 1997; Martin, 2007) in examining the factor structure of RIPLS. In doing this, we used the complementary strengths of both within- 
and between-network construct validation. Within-network validation which is otherwise known as internal construct validation concerns the examination of the measure's factor structure and factor correlation matrix which can be done through reliability analysis and confirmatory factor analysis (CFA). Between-network validation (external construct validation) deals with the examination of how the scale relates with other theoretically-related constructs (Marsh, 1997) which can be done through correlational analysis. The use of two approaches is believed to be more robust and is therefore suggested to be used to complement each other. First, for withinnetwork validity, we performed confirmatory factor analysis (CFA) to examine if our data support the four-factor solution of RIPLS. This approach has been used in a number of validation studies (e.g., Ganotice, Downing, Mak, Chan \& Lee, 2015). Second, we examined the between-network validity of RIPLS by examining its relationship with theoretically relevant constructs: collective team efficacy, team impact on quality of learning, and team impact on clinical reasoning ability. We believe that a potential limitation of the previous validation studies on RIPLS is their failure to establish between-network validity which we also want to establish in this study.

\section{Participants}

Participants of this study consisted of 469 undergraduate students ( $M$ age $=20.95$ years, $S D=1.26$ years, median=21 years) who are pursuing healthcare courses from two Hong Kong Universities and they are predominantly Chinese. They are composed of 191 males (40.7\%) and 262 females (55.9\%). They were from five health care disciplines: Biomedical Sciences, Chinese Medicine, Medicine, Nursing, and Pharmacy; and were distributed from second to fourth year of their programs (Table 1). These students participated in the program called "Interprofessional Team-based Learning for Health Professional Students” (Chan \& Ganotice, 2015a, b). They responded to RIPLS at the beginning of IPTBL session. They have all satisfied the university 
entrance requirement in English language competency as one of the requirements for admission to the various healthcare programs.

\section{Measures}

We utilized the Readiness for Interprofessional Learning Scale with positive and negative dimensions of professional identity (RIPLS, McFadyen et al 2005). The RIPLS measures the following: teamwork and collaboration (9 items, e.g., "Learning with other students will help me become a more effective member of a health care team”), negative professional identity (3 items, e.g., "I don't want to waste my time learning with other healthcare students”), positive professional identity (4 items, e.g., "Shared learning with other healthcare students will help me communicate better with patients and other professionals”), and roles and responsibilities (3 items, e.g., “The function of nurses and therapists is mainly to provide support for doctors”).

Two other measures were used to establish the between-network validity: Students’ Team Experience Questionnaire (TEQ, as cited by Currey, Oldland, Considine, Glanville \& Story, 2015) and Generalized Self-Efficacy Assessment (GSEA, Schwarzer \& Jerusalem, 1995). Specifically, we used three factors of TEQ: overall satisfaction with team experience (e.g., "I have found working as part of the team in my class to be a valuable experience”), team impact on quality of learning (e.g., "I have found being part of the team improved my course grades”), and team impact on clinical reasoning ability ("I have found that being in a team has helped me become better at problem solving”). We used one factor of GSEA: perceived collective efficacy ("I feel confident about the capacity of the group to perform the tasks very well”). TEQ and GSEA were answered on a 5-point Likert scale with higher scores reflecting a greater endorsement of the construct. They have been used in various studies (e.g., Currey et al., 2015; Salanova et al., 2003). 


\section{Statistical analysis}

Confirmatory factor analysis (CFA) was used for the within-network approach to construct validity. CFA is a statistical technique in which an a priori theoretical model is compared with an observed structure in a given data (Milfont \& Fischer, 2010). To examine the validity of the RIPLS, we performed CFA involving four latent variables which underpinned 19 items: teamwork and collaboration (9 items), positive professional identity (4 items), negative professional identity (3 items), and roles and responsibilities (3 items). We did not reverse code students' responses on the three items of negative professional identity to differentiate this subscale from positive professional identity. However, reverse coding is necessary when getting the total readiness scores of the students.

Conventional goodness-of-fit indexes were used in this study: chi-square $\left(\chi^{2}\right)$, chi-square to degrees of freedom ratio ( $\left.\chi^{2} / \mathrm{df}\right)$, root mean square error of approximation (RMSEA), Incremental Fit Index (IFI), Tucker-Lewis Index (TLI), goodness-of-fit index (GFI), normed fit index (NFI), comparative fit index (CFI), normed fit index (NFI), and Akaike information criterion (AIC).

As suggested by Hu and Bentler (1995), the chi square statistic should be nonsignificant. For the normed chi-square, the criteria for acceptance varies from less than 2 to less than 5 (Schumacker \& Lomax, 2004; Ullman, 2001). For RMSEA, values less than .08 indicate acceptable fit. For GFI, NFI, IFI, TLI, and CFI, values higher than .90 show acceptable fit. For the AIC which is used to compare fit when more than one model is estimated, lower values suggest a better fit (Kenny, 2015).

To test the between-network validity of the RIPLS, we clarified that relationships between the four RIPLS factors and various factors of Students' Team Experience Questionnaire: team impact on quality of learning, and team impact on clinical reasoning ability; and Collective 
Efficacy Questionnaire. Statistical Package for Social Sciences (SPSS) version 23 with analysis of moment structures (AMOS) was used for the entire statistical analysis.

\section{Ethical considerations}

The study was completed with the approval of the Human Research Ethics Committee for Non-Clinical Faculties (HRECNCF, reference number EA1507012) of The University of Hong Kong. Student participation was entirely voluntary. The students were assured that the data would be treated with confidentiality and anonymity. Written consent was obtained from the students who decided to participate.

\section{Results}

Preliminary Analyses

Basic statistics were calculated for all the measures. Reliability of the RIPLS factors was acceptable for all the subscales: teamwork and collaboration $(\alpha=.91)$, negative professional identity ( $\alpha=.79)$, positive professional identity $(\alpha=.85)$, and roles and responsibilities $(\alpha=.71$, refer to Table 2).

Within-Network Construct Validation using CFA

Our data support the overall four-factor structure of RIPLS. The fit indexes were adequate: RMSEA=.062, $\mathrm{IFI}=.938, \mathrm{TLI}=.927, \mathrm{GFI}=.911, \mathrm{CFI}=.938, \mathrm{NFI}=.907, \mathrm{AIC}=528.929$. These values indicate a good fit (Byrne, 2010). It was only chi-square $\left(\chi^{2}(146)=406.597, p<.001 ; \chi^{2} / \mathrm{df}=\right.$ 2.785) that was inadequate because a significant $\chi^{2}$ value denotes bad fit. However, the value of the chi-square is directly dependent on sample size suggesting that significant values can be obtained despite trivial discrepancies between the model and the data (Anderson \& Gerbing, 1988; Huang \& Michael, 2000). The standardized factor loadings of the items of original model 
(19 manifest variables) are acceptable except for item number 18 where it only obtained .245 which were then deleted in the subsequent CFA. Stevens (2002) recommended that factor loading which is less than .30 maybe deleted.

After deleting item 18 (“I'm not sure what my professional role will be”), we reran the CFA and the fit was improved after deleting this item: RMSEA=.062, IFI=.942, TLI=.931, CFI=.942, $\mathrm{NFI}=.914, \mathrm{AIC}=456.161$. However, Chi-square was still not adequate: $\left(\chi^{2}(129)=372.161\right.$, $\left.p<.001 ; \chi^{2} / \mathrm{df}=2.885\right)$. To clarify if the original model (19 items, $\left.\chi^{2}=406.597, d f=146\right)$ and the parsimonious model (18 items, $\chi^{2}=372.161, d f=129$ ) are statistically different, we checked the $\chi^{2}$ difference and Akaike's information criterion (AIC). Specifically, the $\chi^{2}$ difference $(17)=34.436$, $p=.007$. The AIC of the parsimonious model is 456.161 smaller than the original model (528.929). Taken together, these findings suggest that the parsimonious model (the reduced 18 items) is a better model.

\section{Between-Network Construct Validation}

The three RIPLS positive factors (e.g., teamwork and collaboration, positive professional identity, and roles and responsibilities) were generally positively related with the positive factors of collaborative work such as overall satisfaction with team-experience, team-impact on quality of learning, team impact on clinical reasoning ability, and collective efficacy ( $r=.13$ to .68). Conversely, the negative professional identity was negatively correlated to all these mentioned variables ( $r=-.18$ to -.36 , refer to Table 2 ). This association of scores on RIPLS with various external variables is consistent with our hypothesis. 


\section{Discussion}

This study was conceptualized to evaluate the validity of the English version of RIPLS using confirmatory factor analysis. The RIPLS has been used in various settings but an examination of its psychometric properties in the Hong Kong context has not been done. In general, results indicated the acceptability of RIPLS when used in the Hong Kong Chinese context after the removal of one item on subscale "roles and responsibilities".

The CFA used to establish the within-network validity indicated that RIPLS has four underlying latent construct: teamwork and collaboration, negative professional identity, positive professional identity, and roles and responsibilities. The various goodness-of-fit indices for this model were acceptable. The factor loadings were all significant at the $p<0.001$ level except for one manifest item (“I am not sure what my professional role will be”) under "roles and responsibilities”. The exclusion of this item in the subsequent analysis improved the reliability of this subscale and the fit indices became even more adequate. This puzzling result may be explained by the modesty norm which is more pronounced in the East (e.g., Cai, Brown, Deng, \& Oakes, 2007). While western individualist culture provides a greater emphasis on self-confidence and self-esteem (Kitayama, Markus, Matsumoto, \& Norasakkunkit, 1997), eastern collectivist culture underscores the modesty norm suggesting the importance of downplaying and/or minimizing of one’s achievements (Kim, Chui, Peng, Cai, \& Tov, 2010). Given this, it is likely that Chinese and other students in a collectivist culture will tend to agree with "I am not sure" items because of prevalence of modesty norm. As a result, the item discrimination index or the ability of the item to discriminate students who are high and low in this item will be problematic because of students' tendency to agree on this item to appear modest. 
To establish the between network validity, we found that scores on the positive factors of RIPLS were positively correlated with the various aspects of team learning: overall satisfaction with team experience, team impact on quality of learning, team impact on clinical reasoning ability and collective ability. For example, teamwork and collaboration was positively correlated with team impact on clinical reasoning ability $(r=.63)$ suggesting that concerted effort in teams is associated with improved group reasoning ability. These results are generally consistent with our theoretical assumption.

A potential limitation of this study is that only predominantly Hong Kong Chinese students were included which limits the generalizability of the results to other Chinese population (e.g., Chinese in Mainland China). It may be necessary to validate RIPLS in a more heterogeneous group of Chinese students. In addition, the range of correlation coefficients was high and some correlation coefficients were low. We invite future researches to pay attention to this to examine if similar pattern of result exist. Despite these limitations, we want to note that this study is important because it serves as a starting point for which research agenda on IPE can be developed to represent the IPE experiences of Asian perspectives in the scholarly conversation on IPE. To our knowledge, this is the first study to examine the acceptability of RIPLS involving predominantly Chinese undergraduate students in Hong Kong. Further, we utilized complementary strengths of within- and between network approaches to construct validity. Given the psychometric acceptability of RIPLS, various studies can be conceptualized with its use. One potential study relates with the readiness of students from various disciplines before and after the IPE sessions. Results of this study can inform evidence-based practices to further the readiness of students to engage in interprofessional activities. 
Generally, our results involving predominantly Chinese students lend support to RIPLS four-factor structure which has been established in other contexts (e.g, McFadyen, Webster, \& Maclaren, 2006; McFadyen et al., 2005; Williams, Brown, \& Boyle, 2012). This does not however undermine the critical observation of scholars on the limitations of RIPLS especially on the theoretical basis for which it was made (e.g., Mahler, Berger, \& Reeves, 2015; Schmitz \& Brandt, 2015). On the positive note, we believe that the non-convergence of results signals a good opportunity for intelligent discussion to arrive at refined version of RIPLS that is informed by theory and has psychometric integrity across culture.

\section{Concluding comments}

On the assumption that the success of students' collaboration depends on their readiness for IPE, it is important that the psychometric properties of RIPLS be established in different cultural settings to draw meaningful results. This study offers evidence that RIPLS is a valid instrument in the Hong Kong Chinese context which can be used in understanding Chinese healthcare students’ readiness for interprofessional learning. As a final note, we hope that this effort to validate RIPLS as a psychological tool can help teachers and researchers in their effort to enrich their research agenda on interprofessional education. The question “Are Chinese undergraduate students ready for shared learning?” can now be answered using the validated RIPLS in the Hong Kong context.

\section{Declaration of interest}

The authors declare no conflicting interest.

\section{References}

Anderson, J. C. \& Gerbing, T. Z. (1988). Structural equation modeling in practice: A review and recommended two-step approach. Psychological Bulletin, 103, 411-423. 
Barr, H. \& Low, H. (2013). Introducing Interprofessional Education. Fareham: Centre for the Advancement of Interprofessional Education (CAIPE).

Byrne, B. M. (2010). Structural equation modelling with AMOS: Basic concepts, applications, and programming (2nd ed.). Mahwah: Erlbaum.

Cai H., Brown J. D., Deng, C., \& Oakes, M. A. (2007). Self-esteem and culture: Differences in cognitive self-evaluations or affective self-regard? Asian Journal of Social Psychology, 10, 162-170.

Chan, L. K. \& Ganotice F. A. (2015a.) The time for Interprofessional Team-based Learning (IPTBL) in Hong Kong: A baseline study. Abstract presented in the Frontiers in Medical and Health Sciences Education, Li Ka Shing Faculty of Medicine, The University of Hong Kong, Hong Kong, 27-28 November 2015, Hong Kong.

Chan, L. K. \& Ganotice, F. A. (2015b). Interprofessional team-based learning checklist: Development and psychometric testing. Abstract presented in the 2015 Frontiers in Medical and Health Sciences Education Conference, Li Ka Shing Faculty of Medicine, The University of Hong Kong, Hong Kong, 27-28 November 2015.

Cloutier, J., Lafrance, J., Michallet, B., Marcoux, L., \& Cloutier, F. (2015). French translation and validation of the Readiness for Interprofessional Learning Scale (RIPLS) in a Canadian undergraduate healthcare student context. Journal of Interprofessional Care, 29 (2), 150155.

Currey, J, Oldland, E, Considine, J., Glanville, D., \& Story, I. (2015). Evaluation of postgraduate critical care nursing students' attitudes to, and engagement with, Team-Based Learning: A descriptive study. Intensive \& Critical Care Nursing, 31(1), 19-28.

Ganotice, F. A., Downing, K., Mak, T., Chan, B., \& Lee, W. Y. (2015). Translation and validation of the Chinese version of Parent-Child Relationship Inventory (PCRI-C) in Hong Kong. Child Indicators Research, 8 (3), 657-670.

Gilbert, J. H. V. (2005). Interprofessional education for collaborative, patient-centred practice. Nursing Leadership, 18 (2), 32-38.

Hambleton, R. K. (2001). The next generation of the ITC test translation and adaptation guidelines. European Journal of Psychological Assessment, 17(3), 164-172.

Hu, L. T., \& Bentler, P. M. (1995). Measuring model fit. In R. H. Hoyle (Ed.), Structural equation modeling: Concepts, issues, and applications. Thousand Oaks: Sage.

Huang C., Michael W. B. (2000). A confirmatory factor analysis of scores on a Chinese version of an academic self concept scale and its invariance across groups. Educational and Psychological Measurement, 60(5), 772-786.

Johnson, D. W., \& Johnson, R. T. (1999). Learning together and alone: Cooperative, competitive, and individualistic learning (5th ed.). Boston, MA: Allyn \& Bacon. 
Kenny, D. A. (2015). Measuring Model Fit. Structural Equation Modelling. Retrieved from http://davidakenny.net/cm/fit.htm.

Kim, Y. H., Chiu C. Y., Peng, S., Cai, H., \& Tov, W. (2010). Explaining East-West differences in the likelihood of making favorable self-evaluations: The role of evaluation apprehension and directness of expression. Journal of Cross-Cultural Psychology, 41, 62-75.

Kitayama, S., Markus, H. R., Matsumoto, H., \& Norasakkunkit, V. (1997). Individual and collective processes in the construction of the self: Self-enhancement in the United States and self-criticism in Japan. Journal of Personality and Social Psychology, 72, 1245-1267.

Lauffs, M., Ponzer, S., Saboonchi, F., Lonka, K., Hylin, U., Mattiasson, A. (2008). Cross-cultural adaptation of the Swedish version of Readiness for Interprofessional Learning Scale (RIPLS). Medical Education, 42 (4), 405-411.

Mahler, C., Giesler, M., Stock, C., Krisam, J., Karstens, S., Szecsenyi, J., \& Krug, K. (2016). Confirmatory factor analysis of the German Readiness for Interprofessional Learning Scale (RIPLS-D). Journal of Interprofessional Care, 30 (3), 381-384.

Maneesriwongul, W., \& Dixon, J. K. (2004). Instrument translation process: A methods review. Journal of Advanced Nursing, 48 (2), 175-186.

Martin, A. (2007). Examining a multidimensional model of student motivation and engagement using a construct validation approach. British Journal of Educational Psychology, 77(Pt 2), 413-444.

Marsh, H. W. (1997). The measurement of physical concept: A construct validation approach. In K. Fox (Ed.), The physical self: From motivation to well-being (pp. 27-58). Champaign, IL: Human Kinetics.

McFadyen, A. K., Webster, V., Strachan, K., Figgins, E., Brown, H., \& McKechnie, J. (2005). The Readiness for Interprofessional Learning Scale: A possible more stable sub-scale model for the original version of RIPLS. Journal of Interprofessional Care, 19 (6), 595 - 603.

McFadyen, A. K., Webster, V. S. \& Maclaren, W. M. (2006). The test-retest reliability of a revised version of the Readiness for Interprofessional Learning Scale (RIPLS). Journal of Interprofessional Care, 20(6), 633 - 639.

Milfont, T. L. \& Fischer, R. (2010). Testing measurement invariance across groups: Applications in cross-cultural research. International Journal of Psychological Research, 3(1), 20112084.

National Research Council (2003). Health Professions Education: A Bridge to Quality. Washington, DC: The National Academies Press.

Nørgaard, B., Draborg, E., \& Sørensen, J. (2016). Adaptation and reliability of the Readiness for Interprofessional Learning Scale in a Danish student and health professional setting. BMC Medical Education, 16 (60). 
Oyarzun, B. A. \& Morrison, G. R. (2013). Cooperative Learning Effects on Achievement and Community of Inquiry in Online Education. Quarterly Review of Distance Education. 14(4), 181-194, 255.

Parsell, G., \& Bligh, J. (1999). The development of a questionnaire to assess the readiness of health care students for interprofessional learning (RIPLS). Medical Education, 33(2), 95100.

Reeves, S., Perrier, L., Goldman, J., Freeth, D., Zwarenstein, M. (2013). Interprofessional education: effects on professional practice and healthcare outcomes (update). Cochrane Database System Review, 23 (2), 124-125.

Salanova, M., Llorens, S., Cifre, E., Martínez, I., \& Schaufeli, W. (2003). Perceived collective efficacy, subjective well-being and task performance among electronic work groups: An Experimental Study. Small Group Research, 34(1), 43-73.

Schumacker, R. E., \& Lomax, R. G. (2004). A beginner's guide to structural equation modeling, Second edition. Mahwah, NJ: Lawrence Erlbaum Associates.

Schwarzer, R. \& Jerusalem, M. (1995). Generalized Self-Efficacy Scale. In: Weinman, J, S Wright, and M Johnson (eds.) Measures in health psychology: A user's portfolio, Causal and control beliefs (pp. 35-37). Windsor England.

Stevens, J. (2002). Applied multivariate statistics for the social sciences. New York: Routledge.

Tamura, Y., Seki, K., Usami, M., Taku, S., Bontje, P., Ando, H., . . Ishikawa, Y. (2012). Cultural adaptation and validating a Japanese version of the readiness for interprofessional learning scale (RIPLS). Journal of Interprofessional Care, 26(1), 56-63.

Tyastuti, D., Onishi, H., Ekayanti, F., \& Kitamura, K. (2014). Psychometric item analysis and validation of the Indonesian version of the Readiness for Interprofessional Learning Scale (RIPLS). Journal of Interprofessional Care, 28 (5), 426-432.

Ullman, J. B. (2001). Structural equation modeling. In B. G. Tabachnick \& L. S. Fidell. Using Multivariate Statistics (4th ed \& pp 653- 771). Needham Heights, MA: Allyn \& Bacon.

Williams, B., Brown, T., \& Boyle, M. (2012). Construct validation of the readiness for interprofessional learning scale: A Rasch and factor analysis. Journal of Interprofessional Care, 26, 326-332. 
Table 1

Participants’ demographics

\begin{tabular}{lcc}
\hline \multicolumn{1}{c}{ Variable } & $\mathrm{N}$ & Percentage (\%) \\
\hline Discipline & 10 & \\
Biomedical Sciences & 13 & 2.10 \\
Chinese Medicine & 203 & 2.80 \\
Medicine & 219 & 43.30 \\
Nursing & 24 & 46.70 \\
Pharmacy & 469 & 5.10 \\
$\quad$ Total & & 100 \\
Year Level & 189 & \\
Second year & 43 & 40.30 \\
Third year & 237 & 9.20 \\
Fourth year & 469 & 50.50 \\
Total & & 100 \\
Age (in years) & 145 & \\
19-20 & 260 & 30.90 \\
21-22 & 39 & 55.40 \\
23-24 & 4 & 8.30 \\
25-26 & 1 & 0.90 \\
> 26 & 20 & 0.20 \\
missing & 469 & 4.30 \\
Total & & 100 \\
Gender & 191 & \\
Male & 262 & 40.70 \\
Female & 16 & 35.90 \\
missing & 469 & 100 \\
$\quad$ Total & & \\
\hline
\end{tabular}


Table 2

Descriptive statistics, internal consistency coefficients, and bivariate correlations among the relevant variables $(\mathrm{N}=469)$

$\begin{aligned} & \text { Within } \text { network measure } \\ & \text { 1. } \begin{array}{l}\text { Teamwork and } \\ \text { collaboration }\end{array} \\ & \text { 2. }\end{aligned}$ Negative professional
identity
3.

